\title{
Astrocytic calcium release mediates peri-infarct depolarizations in a rodent stroke model
}

\author{
Cordula Rakers ${ }^{1}$ and Gabor C. Petzold ${ }^{1,2}$ \\ 'Cerman Center for Neurodegenerative Diseases (DZNE), Bonn, Germany. Department of Neurology, University Hospital Bonn, Bonn, Germany.
}

\begin{abstract}
Stroke is one of the most common diseases and a leading cause of death and disability. Cessation of cerebral blood flow (CBF) leads to cell death in the infarct core, but tissue surrounding the core has the potential to recover if local reductions in CBF are restored. In these areas, detrimental peri-infarct depolarizations (PIDs) contribute to secondary infarct growth and negatively affect stroke outcome. However, the cellular pathways underlying PIDs have remained unclear. Here, we have used in vivo multiphoton microscopy, laser speckle imaging of CBF, and electrophysiological recordings in a mouse model of focal ischemia to demonstrate that PIDs are associated with a strong increase of intracellular calcium in astrocytes and neurons. We found that astroglial calcium elevations during PIDs are mediated by inositol triphosphate receptor type 2-dependent (IP3R2-dependent) release from internal stores. Importantly, Ip3r2-deficient mice displayed a reduction of PID frequency and overall PID burden and showed increased neuronal survival after stroke. These effects were not related to local CBF changes in response to PIDs. However, we showed that the release and extracellular accumulation of glutamate during PIDs is strongly curtailed in Ip3r2-deficient mice, resulting in ameliorated calcium overload in neurons and astrocytes. Together, these data implicate astroglial calcium pathways as potential targets for stroke therapy.
\end{abstract}

\section{Introduction}

Stroke is a leading cause of death and disability and is associated with an enormous socioeconomic burden. In the infarct core, a dramatic reduction of cerebral blood flow (CBF) rapidly triggers cell death. In the tissue surrounding the core - an area called the ischemic penumbra - $\mathrm{CBF}$ is substantially reduced to levels that impair neuronal function, but are high enough to allow for neuronal recovery if $\mathrm{CBF}$ is restored (1). However, the likelihood of neuronal survival and, hence, the chances for an acceptable clinical outcome strongly decline when metabolic demand in the penumbra exceeds the available energy resources. The most prominent example of this scenario is the occurrence of peri-infarct depolarizations (PIDs). These spreading depolarization waves are induced by ionic imbalances in the wake of ischemia (2). Because they impose an additional metabolic burden on the already compromised tissue, PIDs accelerate neurodegeneration and infarct core expansion in experimental models and clinical cases, essentially abrogating the "window of opportunity" for therapeutic interventions in the penumbra $(2,3)$.

Although the exact sequence of events triggering PIDs is not fully established, 2 key molecular mechanisms that take place during PIDs are the accumulation of glutamate in the extracellular space $(4,5)$ as well as elevations of intracellular calcium in astrocytes and neurons (6-8). Glutamate, which can trigger a strong calcium overload in neurons and subsequent neurodegeneration (9), is released by neurons through activation of presynaptic ion channels during PIDs (10), but whether other cell types also contribute to glutamate release has remained unclear. Astroglial calci-

Conflict of interest: The authors have declared that no conflict of interest exists Submitted: July 1, 2016; Accepted: November 3, 2016.

Reference information: / Clin Invest. 2017;127(2):511-516.

https://doi.org/10.1172/JCl89354 um changes can shape synaptic excitability (11) and may mediate some of the CBF changes associated with neuronal activity (12). However, if and how astrocytes contribute to the development of PIDs and whether there is an interplay between astrocytic calcium and glutamate accumulation have remained unknown.

Here, we show that IP3-mediated calcium release from internal stores in astrocytes not only mediates the majority of astroglial calcium changes during PIDs, but also has profound consequences for glutamate accumulation, neuronal calcium overload, PID burden and frequency, as well as neuronal survival and final infarct volume. Our data suggest that modulation of astroglial calcium pathways may represent a novel therapeutic target for further preclinical trials and potentially for stroke therapy.

\section{Results and Discussion}

To identify peri-infarct cortex in mice, we created closed cranial windows in mice and subsequently subjected the animals to permanent middle cerebral artery occlusion (pMCAO). Regional CBF in the cortex under the window was measured before and for 4 hours after $\mathrm{pMCAO}$ induction using laser speckle contrast imaging in predefined cortical regions of interest (ROIs) (Supplemental Figure 1, $\mathrm{A}$ and B; supplemental material available online with this article; https://doi.org/10.1172/JCI89354DS1). On the basis of these measurements, we defined the peri-infarct zone in our model as an area 2.5-3 mm lateral and $1.5 \mathrm{~mm}$ posterior to the bregma, in which CBF consistently remained between approximately $25 \%$ and $35 \%$ after pMCAO (Supplemental Figure 1C). Moreover, we detected the typical CBF signatures of PIDs, which consisted of a period of hypoperfusion followed by transient hyperemia (Supplemental Figure 1D).

Next, we performed multiphoton microscopy of this cortical area to study the cellular dynamics of PIDs. To this end, we labeled cortical astrocytes and neurons with the calcium indicator OGB-1 
A

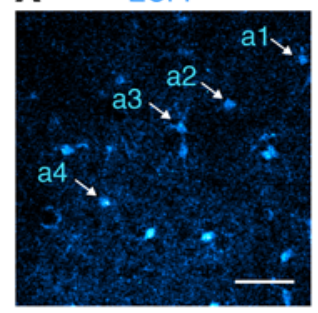

B

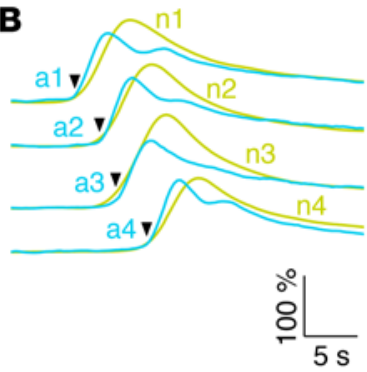

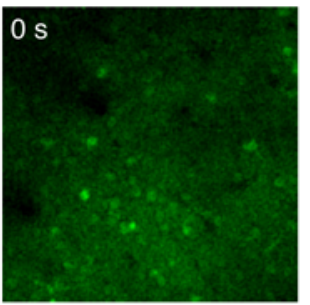

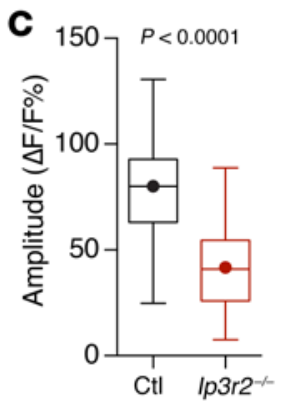

OGB-1 AM
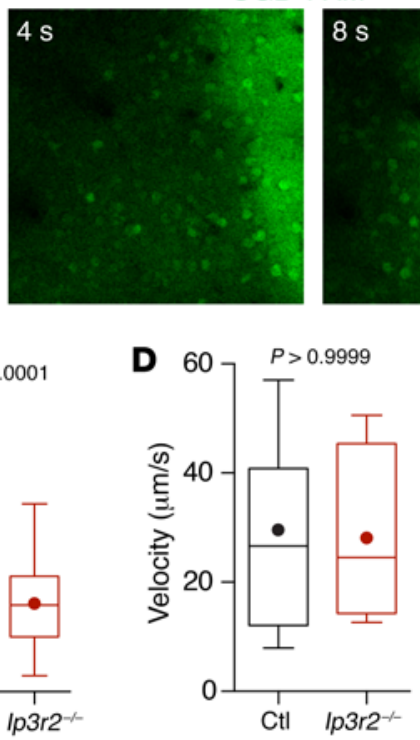
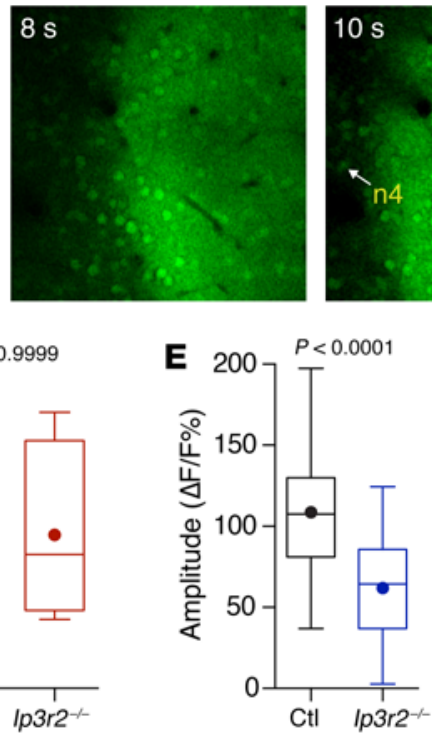

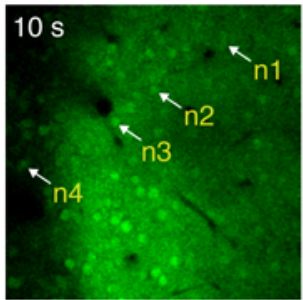

$\mathbf{F}$

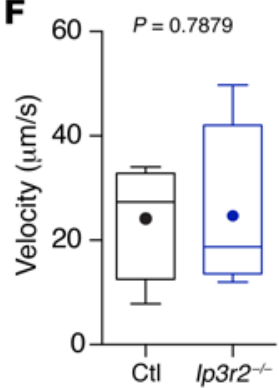

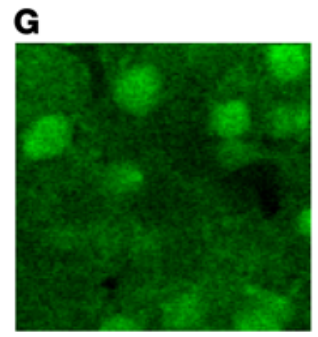

OGB-1 AM

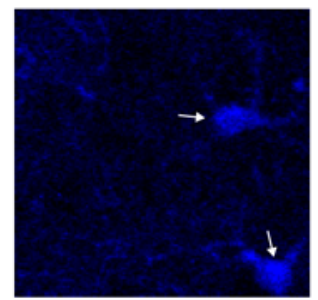

ECFP

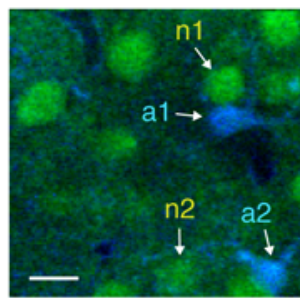

Merge
H

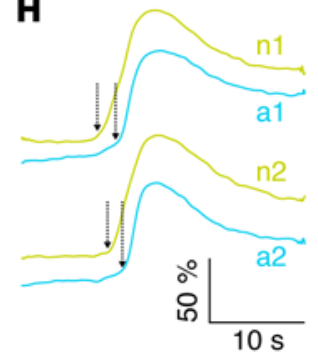

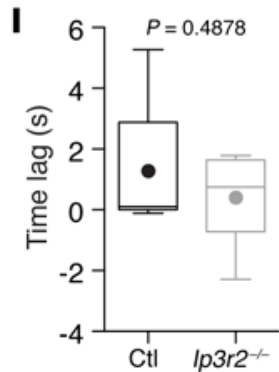

Figure 1. Astrocytic IP3R2 contributes to PID-related calcium elevations. (A and B) OGB-1 AM was injected into peri-infact cortex, and mice expressing ECFP under the $C x 43$ promoter were imaged using multiphoton microscopy. PIDs were identified by typical propagation of cellular calcium transients in neurons (n1-n4) and astrocytes (a1-a4; identified by their ECFP expression). Arrowheads in B indicate propagation. Scale bar: $50 \mu \mathrm{m}$. (C and D) The calcium amplitude in astrocytes from Cx43-ECFP Ip $3 \mathrm{r}^{-1-}$ mice ( $n=117$ cells from 5 mice) was significantly smaller compared with C $x 43-E C F P$ Ip $3 r 2^{+/+}$control mice $(n=147$ cells from 9 mice), while the propagation velocities remained similar. (E and $\mathbf{F}$ ) The amplitude of neuronal calcium transients during PIDs was also reduced in Cx43-ECFP Ip3r2-/- mice compared with controls, while the velocity remained unaltered ( $n=104$ cells from 5 mice vs. $n=142$ cells from 9 mice). (G-I) High temporal and spatial resolution imaging of astrocytes (identified by ECFP expression) and neurons during PIDs showed that astrocytes lagged behind neighboring neurons by $1.29 \pm 0.7$ seconds in $C X 43-E C F P / p 3 r 2^{+/+}$control mice and $0.40 \pm 0.6$ seconds in ECFP Ip3r2 ${ }^{-/-}$mice $(n=8$ neuron-astrocyte pairs from 4 control mice vs. $n=6$ pairs from 3 Ip3r2-/- mice). Scale bar: $10 \mu \mathrm{m}$. Tukey's box-and-whisker plots indicate the median (line), mean (circle), IQR (box), and 1.5 IQR (whiskers) in all figures. All $P$ values were determined by Mann-Whitney $U$ test. Ctl, control; $\Delta \mathrm{F} / \mathrm{F}$, fluorescence intensity relative to baseline.

AM in mice expressing enhanced cyan fluorescent protein (ECFP) under control of the astrocyte-specific connexin 43 promoter (Cx43-ECFP) (13). Blood vessels were labeled with Texas Red dextran (Supplemental Figure 2, A and B, and Supplemental Video 1). Subsequently, we subjected these mice to pMCAO. To verify that the imaging region corresponded to peri-infarct tissue, we determined erythrocyte flux and velocity from line scans along the longitudinal axis of capillaries before and after pMCAO induction (Supplemental Figure 2C). Erythrocyte velocity dropped to $23.3 \%$ $\pm 7.1 \%$ compared with baseline, confirming that our measurements were performed in peri-infarct cortex. Following pMCAO, we detected repetitive PIDs in the imaging region (Figure $1, \mathrm{~A}$ and $\mathrm{B}$ ). As previously reported (6-8), the wave of calcium changes during PIDs involved astrocytes and neurons and propagated through the imaging region (Figure 1, A and B, and Supplemental Video 2).

Next, to identify the origin and role of PID-related calcium elevations in astrocytes, we used mice lacking the IP3 receptor type 2 (Ip3r2---, also known as Itpr $\left.2^{---}\right)(14)$. IP3R2 is the predominant IP3 receptor subtype in astrocytes, but shows negligible expression in neurons (15). To identify astrocytes, we crossed Ip3r2- mice with CX43-ECFP mice. We found that the calcium increase in astrocytes during PIDs was reduced by approximately $50 \%$ in Ip $3 r 2^{-/-}$mice compared with controls (Figure 1C), while the velocity remained unchanged (Figure 1D). Thus, these data identify IP3-mediated release from internal stores as a main component of astroglial calcium changes during PIDs.

Surprisingly, we found that $I p 3 r 2^{---}$mice also showed a strong reduction of the neuronal calcium amplitude during PIDs (Figure $1 \mathrm{E})$, while the neuronal calcium velocity remained unchanged (Figure 1F), indicating that a significant part of the potentially deleterious (9) calcium overload in neurons during PIDs is determined by the amount of calcium released in astrocytes. To investigate the relationship between neuronal and astrocytic calcium, we performed high temporal and spatial resolution imaging and 

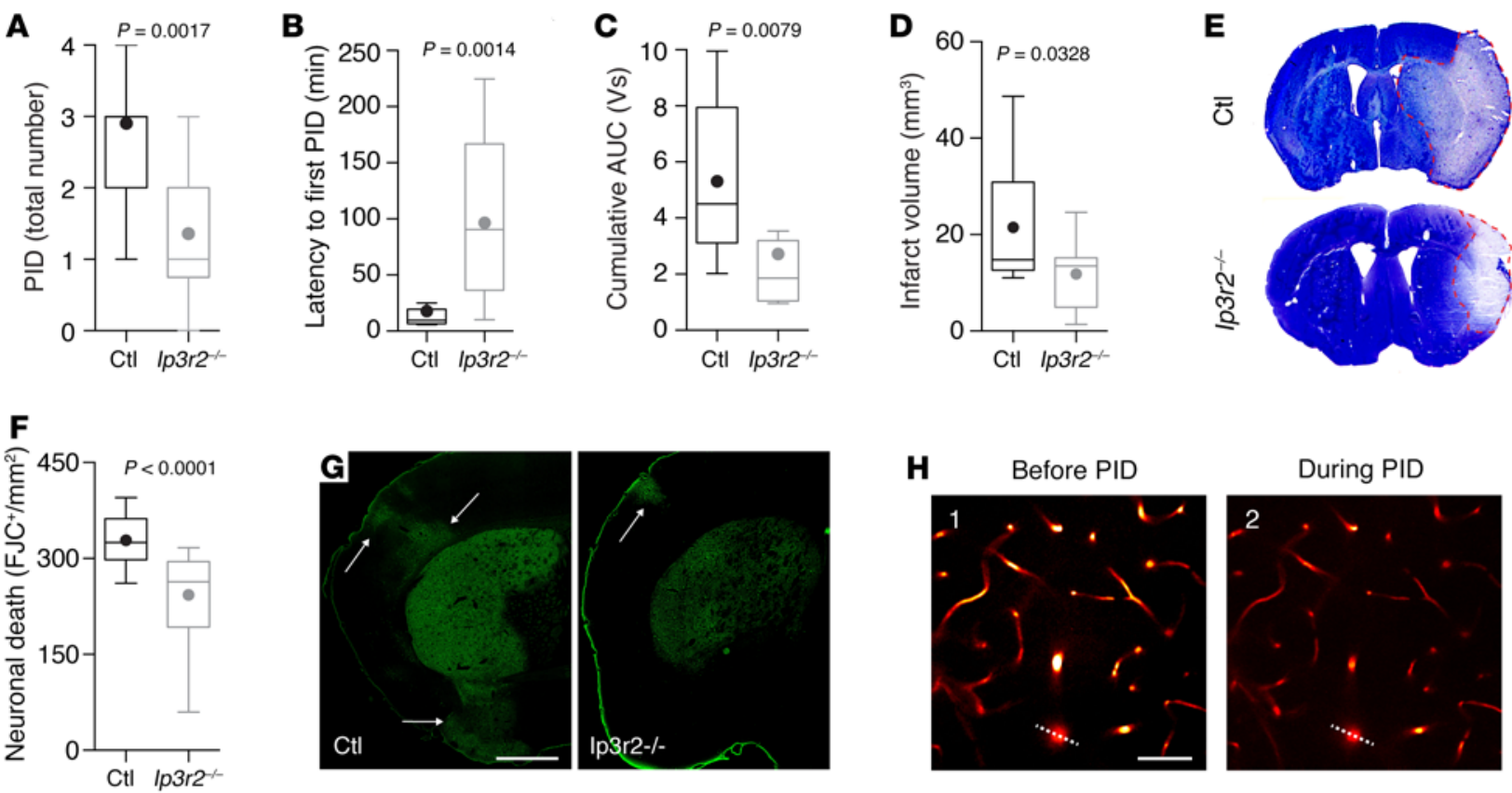

H
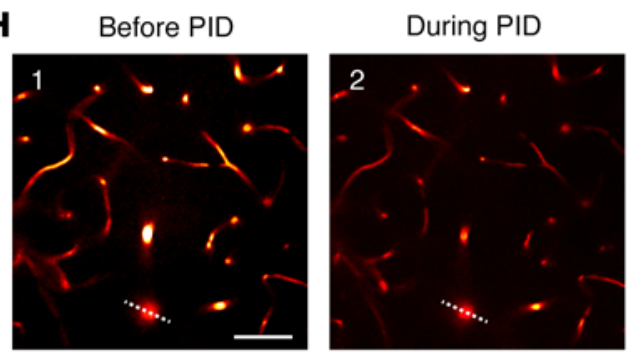

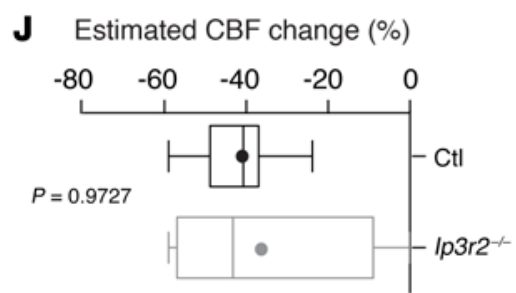

PID-related vasoconstriction

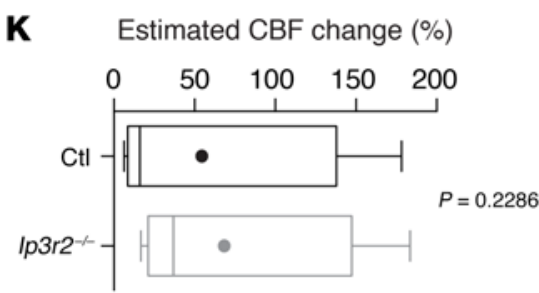

PID-related vasodilation

Figure 2. IP3R2-mediated astroglial signaling reduces PID burden without affecting PID-related cerebrovascular changes. (A-C) Total number of PIDs, latency to first PID, and cumulative AUC as an indicator of overall PID burden were reduced in $1 \mathrm{p} 3 \mathrm{r} 2^{-1-}$ mice $(n=14)$ compared with controls $(n=11$ mice). (D-G) Infarct volumetry and cell densitometry 72 hours after MCAO revealed a reduced infarct size ( $n=15$ mice for each group) and a reduced density of dead neurons (quantified by Fluoro-Jade C [FJC]) ( $n=15$ mice in each group). Arrows in $\mathbf{G}$ indicate cortical neurodegeneration. Scale bar: 1 mm (H) Penetrating arterioles showed strong vasoconstriction during PIDs. Left panel shows the vasculature (labeled with Texas Red dextran) during pMCAO before PID; right panel shows the vasculature during PID. Scale bar: $50 \mu \mathrm{m}$. (I) Resliced image (indicated by the dashed line in $\mathbf{H}$ ) used to determine the arteriolar diameter. Scale bar: $5 \mu \mathrm{m}$. Spatial measurements of Texas Red fluorescence (lower trace) demonstrate the diameter decrease during the PID. (J and K) The vascular response to PID, consisting of a vasoconstriction followed by a variable vasodilation, remained unchanged in both groups ( $n=4$ CX43-ECFP Ip3r2 ${ }^{-/-}$ mice; $n=7$ control mice). All $P$ values were determined by Mann-Whitney $U$ test.

found that the onset of neuronal calcium changes slightly preceded the onset in astrocytes, but that the main signal component in both cell types occurred in synchrony (Figure 1, G-I).

To determine the effects of altered calcium dynamics on overall PID burden, we subjected mice to $\mathrm{pMCAO}$ while measuring the intracortical direct current potential and regional CBF using laser Doppler flowmetry (Supplemental Figure 3, A and B). These experiments showed that the total number of PIDs, PID latency, and cumulative PID burden were significantly reduced in Ip3r2mice (Figure 2, A-C, and Supplemental Figure 3C). Moreover, similar to a different stroke model (16), infarct volume (Figure 2, D and E) and the density of dead neurons (Figure 2, F and G) were both strongly attenuated in $I p 3 r 2^{--}$mice 72 hours after transient $\mathrm{MCAO}$, confirming that the reduced calcium overload in neurons and astrocytes contributed to increased neuronal survival.

Astrocytic calcium changes have also been suggested to mediate vascular responses (12). Therefore, to rule out the possibility that these effects could be attributed to different levels of ischemia or an inherently different susceptibility of Ip $3 r 2^{---}$mice to the initial ischemic depolarization that sets up the brain for subsequent PIDs (3), we measured cortical blood flow following PMCAO as well as the initial depolarization following focal ischemia in a photothrombotic stroke model and found no differences between Ip3r2-- and WT mice (Supplemental Figure 1E and Supplemental Figure 4). Moreover, to rule out the possibility that the PID threshold was influenced by altered CBF dynamics during PID as a consequence of attenuated calcium signaling in Ip $3 r 2^{-}$mice, we determined the response of local cortical arterioles to PIDs. To this end, we labeled the vasculature with Texas Red dextran and measured the diameter of penetrating arterioles, identified by their specific binding of Alexa Fluor 633 (17) (Supplemental Figure 5A). We found that the typical CBF changes during PIDs, which involve prominent vasoconstriction followed by variable vasodilation (Figure 2, H and I) (18), remained unaltered in Ip3r2- 
A
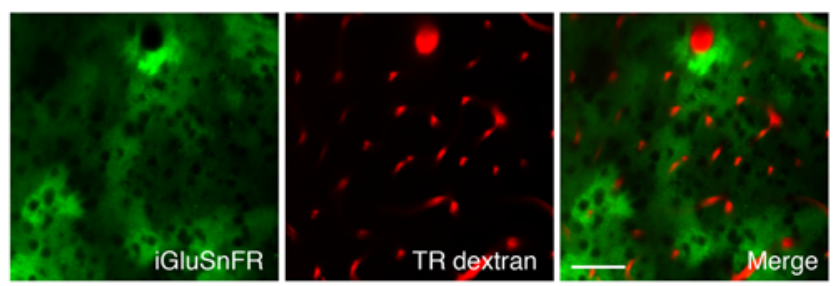

C
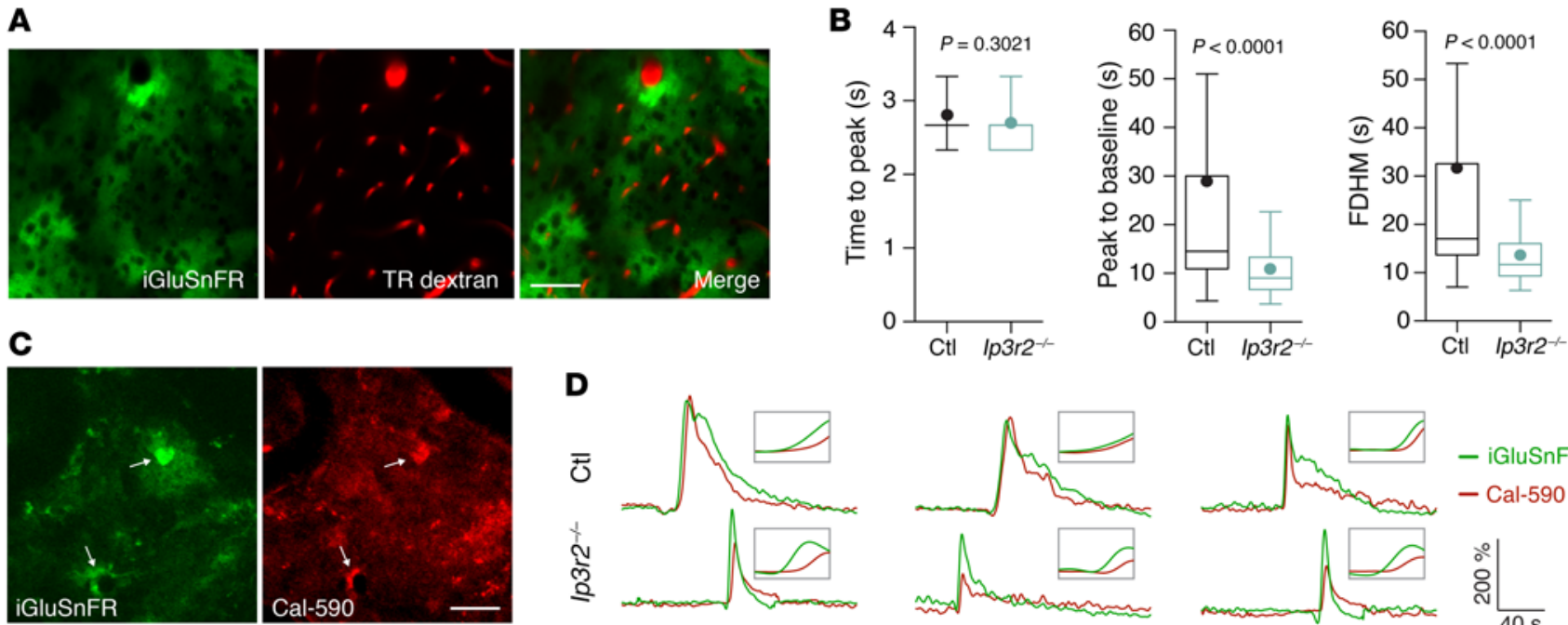

E

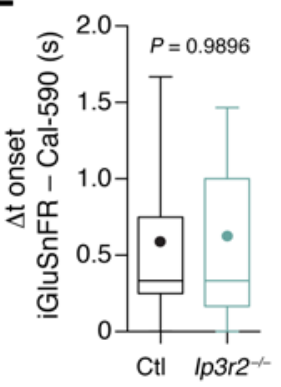

F

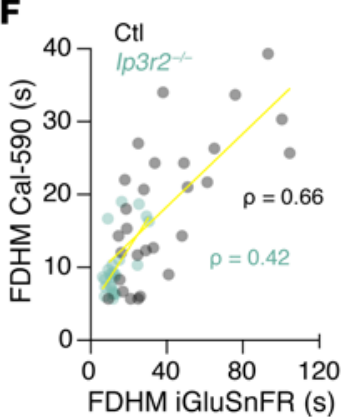

G

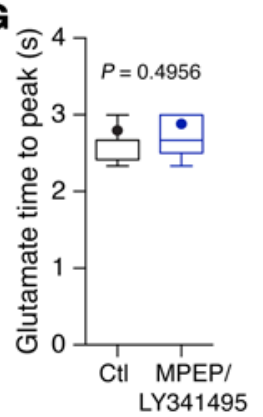

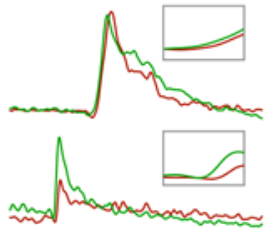

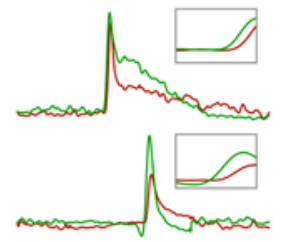

- iGluSnFR - Cal-590

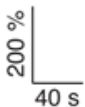

I

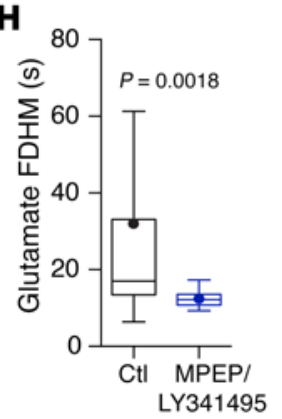

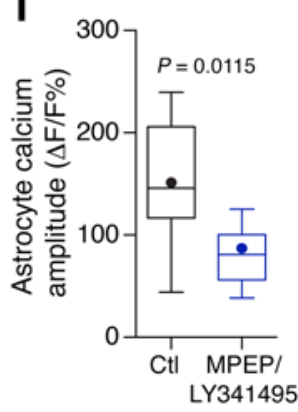

Figure 3. Astroglial IP3R2-mediated signaling contributes to glutamate kinetics during PIDs. (A) An average intensity projection of peri-infarct cortex showed specific and sufficient iGluSnFR expression in cortical astrocytes. The vasculature was visualized with Texas Red (TR) dextran. Scale bar: $50 \mu \mathrm{m}$. (B) There was a strong reduction of the duration of glutamate transients that was mostly caused by an attenuation of the second (sustained) phase of glutamate increase, indicated by a significantly shortened peak-to-baseline time and full duration at half-maximum (FDHM) ( $n=48$ cells from $9 / p 3 r 2^{-/-}$ mice; $n=70$ cells from 10 control mice). (C-E) Simultaneous imaging of glutamate (iGluSnFR) and calcium (Cal-590) in individual astrocytes (arrows) showed that the initial glutamate rise preceded the astroglial calcium transient, but that this calcium transient coincided with the secondary glutamate increase ( $n=25$ astrocytes from $5 \mathrm{lp} 3 \mathrm{r} 2^{-1-}$ mice; $n=28$ astrocytes from 5 control mice). Ip3r2-/- mice showed a shortened glutamate signal and an attenuated calcium rise. $\Delta \mathrm{t}$, time difference. (F) Spearman's rank correlation $(\rho)$ between glutamate and calcium signal durations (FDHM) in overlapping ROls (yellow lines represent linear regression). (G-I) Inhibition of mGluR groups I and II by MPEP/LY341495 (100 $\mu \mathrm{M}$ and $50 \mu \mathrm{M}$, respectively) left the glutamate rise time unchanged, but resulted in significantly shorter FDHM and smaller astroglial calcium amplitudes $(n=28$ cells from 4 mice vs. $n=25$ cells from 4 mice). $P$ values in B, E, and $\mathbf{G}-\mathbf{I}$ were determined by Mann-Whitney $U$ test.

(Figure 2, J and K). Likewise, the cumulative burden of PID-related hypoperfusion was similar (Supplemental Figure 5B), although it may be speculated that the reduced PID frequency may result in a lower burden of hypoperfusion at later time points.

Taken together, our data show that IP3R2-gated calcium release from internal stores strongly contributes to PID frequency in ischemia as well as to cellular calcium overload during each PID, ultimately resulting in decreased infarct volume and increased neuronal survival. To gain a better understanding of how astrocytic calcium may mediate these effects, we explored the kinetics of glutamate, which can be modulated by astrocytes (19) and is released during spreading depolarizations $(4,5)$. To this end, we intracortically injected adeno-associated virus (AAV) encoding the fluorescent glutamate indicator iGluSnFR (20) under the astrocyte-specific glial fibrillary acidic protein (GFAP) promoter 2 weeks before PMCAO. This resulted in robust expression of iGluSnFR by astrocytes (Figure 3A and Supplemental Figure 6, A and B).
PIDs were accompanied by a transient and propagating increase in iGluSnFR fluorescence (Supplemental Figure 6C), indicating glutamate release and diffusion near astrocytic surfaces. Near-astrocyte glutamate kinetics consisted of 2 components: a fast peak followed by a delayed slow rise (Supplemental Figure 6C). We compared PIDs in Ip3r2- ${ }^{--}$and WT control mice and found that the glutamate signals were significantly shorter in Ip $3 \mathrm{r}^{--}$mice (Figure 3B) . This difference was due to a shortening of the delayed slow-rise component of the glutamate signal, indicated by faster decay times than those in control mice (Figure $3 \mathrm{~B})$. To better understand the dynamics between astrocytic calcium elevations and glutamate, we labeled peri-infarct cortex of mice transfected with AAV-GFAP-iGluSnFR with the red-shifted calcium indicator Cal-590 (21), which resulted in colabeling of individual astrocytes with iGluSnFR and Cal-590 (Figure 3C). Our analysis of calcium and glutamate dynamics in individual astrocytes during PIDs revealed that the fast component of the 
iGluSnFR signal preceded astroglial calcium transients (Figure 3, D and E). This finding was consistent with our high-resolution imaging data (Figure 1, G-I) and indicated that the first component of the glutamate signal detected by astrocytic iGluSnFR may represent neuronal glutamate that induces a calcium rise in astrocytes, although iGluSnFR detects a mixture of neuronal and astroglial glutamate (22), and we cannot exclude the possible influence of different indicator kinetics. In turn, this calcium increase coincided with the delayed slower component of the glutamate signal (Figure 3D), and the durations of both signals were strongly correlated (Figure 3F), indicating a secondary glutamate release phase associated with astroglial calcium. Consequently, the attenuated calcium rise in $\mathrm{Ip} 3 \mathrm{r} 2^{-/-}$mice was also correlated with a shortened glutamate signal (Figure 3, D-F), suggesting that the effects of astroglial IP3-mediated calcium signaling on neuronal calcium, PID threshold, and infarct volume may be mediated by modulation of glutamate release during PIDs. To further test this hypothesis and investigate the potential for pharmacological intervention, we topically applied a combination of drugs that inhibit the effects of neuronal glutamate on astroglial metabotropic glutamate receptors (22). This resulted in shortened glutamate decay times and strongly reduced astroglial calcium amplitudes during PIDs (Figure 3, G-I), supporting the notion that astroglial calcium contributes to secondary glutamate release.

In conclusion, these data suggest that by contributing to extracellular glutamate accumulation, IP3R2-mediated calcium release from astrocytic internal stores is a major determinant of PID burden, neuronal calcium overload, and ischemic neurodegeneration. Interestingly, IP3-gated calcium release from the endoplasmic reticulum is a trigger of glutamate release from astrocytes (19), although the exact mechanisms remain under debate (23). However, our data suggest that astrocytes are an important source of glutamate during PIDs and that this mechanism has previously unrecognized, yet profound effects on the frequency and overall burden of these deleterious depolarization waves. Hence, interference with these pathways may potentially lead to the development of novel treatment strategies to ameliorate PID-induced damage in acute brain disorders.

\section{Methods}

Further information can be found in the Supplemental Methods.

Statistics. Comparisons between 2 groups were conducted using a 2-sided Mann-Whitney $U$ test. Correlation was calculated as Spearman's rank correlation. All data were analyzed using GraphPad Prism 6 (GraphPad Software) and are expressed as the mean \pm SEM or as Tukey's box-and-whisker plots indicating the median, mean, interquartile range (IQR), and 1.5 IQR. A $P$ value of less than 0.05 was considered statistically significant. Six animals were excluded from the analysis (one control mouse died during an imaging experiment; three $I p 3 r 2^{---}$and two control mice died after MCAO before the infarct volumetry endpoint).

Study approval. All experiments were performed according to animal care guidelines and approved by the Landesamt für Natur, Umwelt und Verbraucherschutz of North Rhine-Westphalia (Germany).

\section{Author contributions}

GCP conceptualized and supervised the study. CR performed the experiments. Both authors established the methodology, performed the data analysis, and wrote the manuscript.

\section{Acknowledgments}

We thank Jan Peter, Theresa Hundt, Armin Keller, Patricia Schmidbauer, Marco Foddis, Christoph Moehl, Kevin Keppler, and Andrea Delekate for their technical assistance; Ju Chen (UCSD, San Diego, California, USA) for sharing Ip3r2 $2^{-1-}$ mice; and Martin Theis (Bonn University, Bonn, Germany) for sharing Cx43-ECFP mice. This work was supported by grants to GCP from the Else Kröner-Fresenius Foundation; the German Science Foundation DFG (PE1193/21); the DZNE and Canadian Institutes of Health Research (CIHR) through the CoEN Initiative; the European Union (EU) Joint Programme - Neurodegenerative Disease Research (JPND) program (EU Horizon 2020 Research and Innovation Program, grant agreement 643417/DACAPO-AD); and the DZNE.

Address correspondence to: Gabor C. Petzold, German Center for Neurodegenerative Diseases (DZNE), Sigmund-Freud-Str. 27, 53127 Bonn, Germany. Phone: 49.228.28751625; E-mail: gabor. petzold@dzne.de.
1. Hossmann KA. Viability thresholds and the penumbra of focal ischemia. Ann Neurol. 1994;36(4):557-565.

2. Lauritzen M, Dreier JP, Fabricius M, Hartings JA, Graf R, Strong AJ. Clinical relevance of cortical spreading depression in neurological disorders: migraine, malignant stroke, subarachnoid and intracranial hemorrhage, and traumatic brain injury. JCereb Blood Flow Metab. 2011;31(1):17-35.

3. Dreier JP, Reiffurth C. The stroke-migraine depolarization continuum. Neuron. 2015;86(4):902-922.

4. Fabricius M, Jensen LH, Lauritzen M. Microdialysis of interstitial amino acids during spreading depression and anoxic depolarization in rat neocortex. Brain Res. 1993;612(1-2):61-69.

5. Enger R, et al. Dynamics of ionic shifts in cortical spreading depression. Cereb Cortex. 2015;25(11):4469-4476.

6. Duffy S, MacVicar BA. In vitro ischemia promotes calcium influx and intracellular calcium release in hippocampal astrocytes. J Neurosci. 1996;16(1):71-81.

7. Murphy TH, Li P, Betts K, Liu R. Two-photon imaging of stroke onset in vivo reveals that NMDA-receptor independent ischemic depolarization is the major cause of rapid reversible damage to dendrites and spines. J Neurosci. 2008;28(7):1756-1772.

8. Chuquet J, Hollender L, Nimchinsky EA. High-resolution in vivo imaging of the neurovascular unit during spreading depression. J Neurosci. 2007;27(15):4036-4044.

9. Bano D, Nicotera P. Ca2+ signals and neuronal death in brain ischemia. Stroke. 2007; 38(2 Suppl):674-676.

10. Pietrobon D, Moskowitz MA. Chaos and commotion in the wake of cortical spreading depression and spreading depolarizations. Nat Rev Neurosci. 2014;15(6):379-393.

11. Khakh BS, Sofroniew MV. Diversity of astrocyte functions and phenotypes in neural circuits. Nat Neurosci. 2015;18(7):942-952.

12. Petzold GC, Murthy VN. Role of astrocytes in neurovascular coupling. Neuron. 2011;71(5):782-797.

13. Degen J, et al. Dual reporter approaches for identification of Cre efficacy and astrocyte heterogeneity. FASEB J. 2012;26(11):4576-4583.

14. Li X, Zima AV, Sheikh F, Blatter LA, Chen J. Endothelin-1-induced arrhythmogenic Ca2+ signaling is abolished in atrial myocytes of inositol-1,4,5-trisphosphate(IP3)-receptor type 2-deficient mice. Circ Res. 2005;96(12):1274-1281.

15. Sharp AH, et al. Differential cellular expression of isoforms of inositol 1,4,5-triphosphate receptors in neurons and glia in brain. JComp Neurol. 1999;406(2):207-220.

16. Li H, Xie Y, Zhang N, Yu Y, Zhang Q, Ding S. Disruption of $\mathrm{IP}_{3} \mathrm{R} 2$-mediated $\mathrm{Ca}^{2+}$ signaling pathway in astrocytes ameliorates neuronal death and brain damage while reducing behavioral 
deficits after focal ischemic stroke. Cell Calcium. 2015;58(6):565-576.

17. Shen Z, Lu Z, Chhatbar PY, O'Herron P, Kara P. An artery-specific fluorescent dye for studying neurovascular coupling. Nat Methods. 2012;9(3):273-276.

18. Shin HK, Dunn AK, Jones PB, Boas DA, Moskowitz MA, Ayata C. Vasoconstrictive neurovascular coupling during focal ischemic depolarizations.
JCereb Blood Flow Metab. 2006;26(8):1018-1030.

19. Parpura V, Grubišić V, Verkhratsky A. Ca(2+) sources for the exocytotic release of glutamate from astrocytes. Biochim Biophys Acta. 2011;1813(5):984-991.

20. Marvin JS, et al. An optimized fluorescent probe for visualizing glutamate neurotransmission. Nat Methods. 2013;10(2):162-170.

21. Tischbirek C, Birkner A, Jia H, Sakmann B, Kon- nerth A. Deep two-photon brain imaging with a red-shifted fluorometric $\mathrm{Ca} 2+$ indicator. Proc Natl Acad Sci U S A. 2015;112(36):11377-11382.

22. Haustein MD, et al. Conditions and constraints for astrocyte calcium signaling in the hippocampal mossy fiber pathway. Neuron. 2014;82(2):413-429.

23. Araque A, Carmignoto G, Haydon PG, Oliet SH, Robitaille R, Volterra A. Gliotransmitters travel in time and space. Neuron. 2014;81(4):728-739. 